\title{
PROKLA-Redaktion
}

\section{Editorial: Finanzialisierung, Konzentration, veränderte Unternehmensformen}

Im Jahre 1998 gab bereits einmal ein PROKLA-Heft zu Konzentration (PROKLA 113; Konzentration, Internationalisierung, Vernetzung). Damals stand die Fusion der Konzerne Daimler und Chrysler an, die Daimler-ChefJürgen Schrempp euphorisch als „Hochzeit, die im Himmel geschlossen wird" bezeichnete. Neun Jahre später resümierte die Süddeutsche Zeitung, es habe sich wohl eher um eine „Hochzeit des Grauens" gehandelt Die Großmachtphantasie Schrempps hat den Konzern ca. 40 Mrd. Euro gekostet. Das PROKLA-Heft von 1998 widmete sich jedoch nicht in erster Linie einzelnen megamergers, sondern der historischen wie der aktuellen Entwicklung der Konzentrationsprozesse. Industrielle Restrukturierung folgte, so war zu dieser Zeit zu diagnostizieren, häufig der Leitlinie der Konzentration auf die „Kernkompetenzen“, was damit verbunden war, dass etwa in der Chemie- und Pharmaindustrie oder in der Computerindustrie nationale oder globale Netzwerke aufgebaut wurden, innerhalb derer die großen Konzerne zentrale Machtpositionen bei der Organisation von Forschung und Entwicklung wie bei der Produktion einnahmen.

Anders als vielfach prophezeit wurde, hat sich inzwischen noch deutlicher als Mitte der 1990er Jahre gezeigt, dass es keinen linearen Trend einer ständig zunehmenden "Monopolisierung" gibt, sondern eine Vielzahl von widersprüchlichen Erscheinungen. Wie bereits die historische Analyse von
Leslie Hannah in PROKLA 113 zeigte, heißt für ein Unternehmen „einmal groß“ keineswegs: „für immer groß“. Von den 200 international größten Unternehmen des Jahres 1912 existierte Mitte der 1990er Jahre nur noch die Hälfte. Ähnliches ist festzustellen, wenn man die Liste der umsatzstärksten westdeutschen Unternehmen des Jahres 1978 mit derjenigen des Jahres 2011 vergleicht: Befanden sich damals die Firmen Opel und Ford noch unter den ersten 20 , so sind sie heute nicht einmal mehr auf der Liste der ersten 100. Andere Spitzenreiter des Jahres 1978 existieren überhaupt nicht mehr, sondern wurden als Konzerne gänzlich zerschlagen: Hoechst (1978: Platz 5), AEG Telefunken (1976: Platz 9) und Mannesmann (1976: Platz 11) (Wirtschaftswoche, Nr. 30/1979: 72). Und auch auf der internationalen Ebene haben sich die Verhältnisse deutlich gewandelt. Dominierten bis vor einigen Jahren noch US-amerikanische und westeuropäische Unternehmen entsprechende Listen, so finden sich in der Forbes-Liste der „Leading Companies“ von 2011 unter den 20 weltweit umsatzstärksten Unternehmen inzwischen zwei aus der VR China (Sinopec-China Petroleum: Platz 4, PetroChina: Platz 6), eines aus Brasilien (Petrobras-Petróleo Brasil: Platz 16) und eines aus Südkorea (Samsung: Platz 20). Gleichzeitig sind auch die Dimensionen von Größe andere geworden. Stand General Motors 1971 mit einem Umsatz von 30.435 Mio. Dollar an der Spitze aller US-Unternehmen, 
so landete es 2011 mit einem fast fünf Mal so großen Umsatz von 150.300 Mio. Dollar auf Platz 5 der entsprechenden Liste (www. forbes.com/global/2000, Wirtschaftswoche, Nr. 22/1973: 96).

Das Wachstum der großen Konzerne kann durch eine Ausdehnung der bisherigen Aktivitäten und Geschäftsfelder erfolgen, oder auch durch den Erwerb von anderen Unternehmen. Gab es im Deutschland der 1920er und 1930er Jahre einige spektakuläre Großfusionen im Bereich von Eisen und Stahl sowie im Chemiesektor, so handelte es sich in der Nachkriegszeit meist eher darum, dass kleinere Firmen von ihren großen Konkurrenten übernommen wurden. In den letzten 20 Jahren kam es jedoch wieder vermehrt zu „Elefantenhochzeiten“ nach dem Muster von DaimlerChrysler. Eine solche stellte z.B. 1999 die Fusion der Stahlkonzerne Thyssen und Krupp dar. Im Sommer 2012 gingen Gerüchte über eine geplante weitere Fusion des mittlerweile erheblich angeschlagenen Stahlkonzerns ThyssenKrupp (Umsätze: Platz 18) mit Siemens (Umsätze: Platz 4) durch die Wirtschaftspresse, wurden aber bisher zumindest von ThyssenKrupp heftig dementiert. Im Bereich der Finanzdienstleistungen übernahm 2001 die Allianz Versicherung die zweitgrößte deutsche Bank, die Dresdner Bank, die 2008 wiederum an die Commerzbank (zuvor drittgrößte Bank) weiter verkauft wurde (Handelsblatt, 30.7.2012).

Der immer wieder verkündete tiefere Sinn derartiger Fusionen liegt vorrangig in „Synergieeffekten“ oder - wie der spätere Daimler-Chef Dieter Zetsche 2006 etwas offener erklärte - in der „Vermeidung von Doppelarbeit". Dazu sollten im DaimlerChrysler-Fall nach ersten Plänen 16.000 Stellen gestrichen werden, faktisch waren es dann 9.300. Ähnlich sah es für die Arbeitnehmer/innen bei zahllosen anderen Fusionen aus. Auch der Zusammenschluss von
Commerzbank und Dresdner Bank hatte zum Ziel, hunderte von Filialen zu schließen und 9.000 Arbeitsplätze zu streichen. Mergers bedeuten somit für die einen den Verlust des Arbeitsplatzes, für die anderen mehr und intensivere Arbeit (www.manager-magazin. de, 20.12.2005; Handelsblatt, 1.12.2010).

Selbst wenn sich auf diese Art Einsparungen bei den Lohnkosten ergeben, ist keineswegs ausgemacht, dass Fusionen insgesamt wirtschaftlich erfolgreich sind. So haben zahlreiche Studien gezeigt, dass in den allermeisten Fällen weder die Umsätze noch die Gewinne und der Börsenwert der neu entstandenen Unternehmen steigen, sondern häufig sogar zurückgehen, die performance der Unternehmen also „enttäuscht“. Immer wieder wird festgestellt, dass Organisationsprinzipien, Entlohnungssysteme und erst recht die IT-Systeme nicht zusammen passen, so dass erhebliche Reibungsverluste entstehen (Wirtschaftswoche, Nr. 31/2008: 60f). Insofern war das DaimlerChryslerDebakel keineswegs einzigartig. Auch Schrempps Vorgänger Edzard Reuter hatte sich bereits ein Jahrzehnt zuvor bei seiner Vision, einen ,integrierten Technologiekonzern" zu schaffen, erheblich verkalkuliert. Die große Einkaufstour wurde ein spektakulärer finanzieller Misserfolg. Die von einigen großen Konzernen über lange Zeit verfolgten Projekte einer "Welt AG"verloren seither an Beliebtheit, dafür entstanden andere, bisher unbekannte Konstellationen.

Eine der neuen Entwicklungen der Jahre nach 2000 besteht darin, dass bei mergers \& acquisitions vermehrt Konzerne aus Schwellenländern als Käufer auf den Plan treten und in manchen Fällen sogar Unternehmen ihrer früheren Kolonialherren aufkaufen, wie die indische Tata Motors, die 2008 die britischen Traditionsmarken Jaguar und Land Rover übernahmen. Unternehmen der VR China gaben allein in den ersten drei Monaten des Jahres 2012 nicht weniger als 
700 Mio. Dollar aus, um deutsche Firmen aufzukaufen, wie z.B. den Weltmarktführer im Geschäft mit Betonpumpen, Putzmeister (www.manager-magazin.de, 26.3.2008).

Eine weitere Neuerung bestand in den unterschiedlich erfolgreichen - Versuchen von großen Unternehmen, noch sehr viel größere zu übernehmen. Vodafone, ein britischer Mobilfunkbetreiber mit rd. 7.000 Beschäftigten, zog den deutschen Traditionskonzern Mannesmann mit seinen 130.000 Beschäftigten in eine Übernahmeschlacht hinein, die bis heute als weltweit teuerster Zukauf gilt. Der deutsch-britische Mega-Deal ist nicht allein dadurch bekannt geworden, dass er mit dubiosen Abfindungen in Millionenhöhe an führende Manager von Mannesmann einherging. Er zeigte auch, welche Summen für diejenigen anfallen, die das Geschäft finanziell vorbereiten und begleiten: Goldman \& Sachs und UBS Warburg Dillon Read sollen für ihre Beratungsleistungen jeweils rd. 75 Mio Dollar Honorar kassiert haben. Solche Honorare fließen unabhängig davon, ob die Fusionen sich später als rentabel erweisen oder nicht. Die Investmentfirmen haben daher ebenso ein Interesse daran, die Kuh, die ihnen Milch gibt, weiter zu füttern, wie die Rechtsanwälte und Ratingfirmen, die solche Prozesse initiieren und begleiten. Ein ähnlicher Versuch von Porsche, die Nr. 1 der deutschen Automobilbranche, VW, mit Hilfe von riesigen Krediten zu übernehmen, scheiterte hingegen. Die Transaktion lief im Sommer 2012 letztlich genau anders herum, wobei es VW durch einen Trick gelang, den Kauf der Porsche AG mit ihren rd. 15.000 Beschäftigten als „Umstrukturierung“ zu deklarieren, so dass er praktisch steuerfrei blieb (www.spiegelonline.de, 4.2.2000, Handelsblatt, 9.6.2012).

Trotz allen Drucks durch die an Geschäften interessierten Berater und das journalistische Umfeld können diese das
Fusionsgeschehen nur begrenzt beeinflussen, da die Erwartungen fusionswilliger Unternehmen letztlich davon abhängen, wie sie die Entwicklungen auf verschiedenen Märkten einschätzen. Die Zahlen der jährlichen Fusionen schwanken daher, bei insgesamt anhaltendem Aufwärtstrend, erheblich. Geht man von den in Deutschland beim Bundeskartellamt angemeldeten Fusionsvorhaben aus, so stiegen diese seit 1990 vor allem rund um das Jahr 2000 und erreichten 2007 einen Höchststand, um im folgenden Jahr - parallel zur einsetzenden Finanzmarktkrise - stark zurück zu fallen. Dabei ließ sich eine verstärkte Tendenz zu fremdfinanzierten Übernahmen (leveraged buyouts) sowie zu größeren, kostspieligeren und riskanteren Übernahmen registrieren. Seitdem hat sich die Lage weiterhin höchst unübersichtlich entwickelt. Zwar nahm die Zahl der Fusionen wieder zu, und der Markt für Käufe und Verkäufe von Unternehmen scheint lebhaft wie eh und je. Doch das Handelsblatt stellte im Dezember 2011 auch fest, die Zeiten für Zusammenschlüsse seien schlecht und die Fälle häuften sich, bei denen geplante Vorhaben - wie etwa die Megafusion von Deutscher Börse und New York Stock Exchange - nicht zustande kämen, weil entweder Aufsichtsbehörden intervenierten oder aber die Märkte „unberechenbar“ geworden seien (Handelsblatt, 20.12.2011).

Ganz neu sind Erkenntnisse über die Wechselhaftigkeit des Geschehens bei der Zusammenführung oder dem Auseinanderbrechen von Unternehmen allerdings nicht. Im ersten Band des Kapital setzte sich Marx auch mit dessen längerfristigem Wachstum auseinander. Mit dem „gesellschaftlichen Reichtum“würden einzelne Kapitale größer - was Marx „Konzentration“ nennt - und gleichzeitig käme es zu einem Umverteilungsprozess innerhalb der Kapitalisten, insbesondere zur „Zentralisation“ einzelner 
Kapitale, also zur „Konzentration bereits gebildeter Kapitale, Aufhebung ihrer individuellen Selbständigkeit, Expropriation von Kapitalist durch Kapitalist, Verwandlung vieler kleineren in weniger größere Kapitale... Das Kapital schwillt hier in einer Hand zu großen Massen, weil es dort in vielen Händen verloren geht." (MEW 23: 654). Nüchtern diagnostizierte Marx: „Je ein Kapitalist schlägt viele tot." (Ebd.: 790) Dennoch handelt es sich Marx zufolge um einen Prozess, der nicht nur eine Richtung kennt: den Tendenzen zur Zusammenballung stünden solche der Zersplitterung gegenüber: ,... das Wachstum der funktionierenden Kapitale ist durchkreuzt durch die Bildung neuer und die Spaltung alter Kapitale.“ (Ebd.: 654) Marx geht davon aus, dass die Konkurrenz den individuellen Kapitalisten zwinge, „sein Kapital fortwährend auszudehnen, um es zu erhalten, und ausdehnen kann er es nur vermittelst progressiver Akkumulation" (ebd.: 618).Es geht ihm dabei nicht nur um die economy of scale (ebd: 654f), sondern auch darum, sich den Herausforderungen der „beständigen Revolutionen in den Produktionsmethoden selbst“ zu stellen und die „damit beständig verknüpfte Entwertung von vorhandnem Kapital, den allgemeinen Konkurrenzkampf und die Notwendigkeit, die Produktion $\mathrm{zu}$ verbessern und ihre Stufenleiter auszudehnen, bloß als Erhaltungsmittel und bei Strafe des Untergangs" zu bestehen (MEW 25: 254f). Konzentration des Kapitals ist so gesehen ein 'normaler', notwendiger Prozess der Reproduktion des Kapitals. Zum Problem wird er, wenn er zum Monopol führt und die Konkurrenz negiert. Dieser Prozess, so Marx, „fordert daher die Staatseinmischung heraus" (ebd.: 454).

Das Kapital kann also um des Kapitalismus willen nicht sich selbst überlassen bleiben. Der Staat muss intervenieren. Und das tut er seit jeher, aber mit signifikanten
Unterschieden. Historisch betrachtet waren es zunächst andere Kapitalfraktionen, auch massenhaft betroffene Bürger/innen, die sich gegen Monopolpreise strategisch platzierter Unternehmen wehrten; so in den USA bereits in den 1880er Jahren, z.B. wegen zu hoher Frachttarife der Eisenbahngesellschaften. Daraufhin wurde 1890 das erste Antitrustgesetz (Sherman Act) erlassen, später durch weitere Gesetze ergänzt bzw. verschärft. Das führte z.B. zur Zerschlagung des Standard Oil-Konzerns (1911), der damals ein Monopol auf das noch dominante Leuchtmittel Petroleum hatte oder zur Auflösung der Telefongesellschaft AR\&T (1982).

In vielen Industriestaaten wurden ähnliche Gesetze gegen Monopolisierungstendenzen erlassen, aber sehr unterschiedlich angewendet. Ihre Bedeutung wandelte sich zudem je nach der ökonomisch relevanten Bezugsebene, z.B. relativierte sich nationale Marktmächtigkeit nach Bildung der Europäischen Union auf der europäischen bzw. auf der Weltmarktebene. Zuweilen waren bestimmte Monopole auch politisch gewünscht und gesellschaftlich akzeptiert, z.B. bei Dienstleistungen im Infrastrukturbereich (Schienenverkehr, Post etc.). Grundüberzeugung auch linker Parteien war es seit jeher, dass die Gewährleistung der Funktionen des Gemeinwesens und das bezieht die Basisleistungen im Bereich von Kommunikation, Verkehr, Gesundheit Wohnen etc. ein - staatlich garantiert, d.h. allgemein zugänglich und für jedermann erschwinglich sein müssen. Demgegenüber bleiben die Wirkungen von Privatisierungen und Ausdehnung der privaten Konkurrenz in den letzten beiden Jahrzehnten umstritten (das schlagendste Negativbeispiel stellt die Privatisierung der Britischen Eisenbahngesellschaft dar).

Moderne Industriestaaten haben oftmals eine hohe Toleranz gegenüber 
Konzentrationsprozessen in der privaten Wirtschaft aufgewiesen. Das gilt besonders für die ökonomisch geschwächte Weimarer Republik, in der krisenbedrohte Unternehmen in einigen Wirtschaftsbereichen meinten, nur in einem Verbund überleben zu können. Das lässt sich aber auch in der Gegenwart aufzeigen. Die dominierende Freihandelsorientierung in den USA akzeptierte die Schließung bzw. Abwanderung weiter Teile des Verarbeitenden Gewerbes in Niedriglohnländer mit drei bemerkenswerten Ausnahmen: Luft-und Raumfahrtindustrie, Rüstungsindustrie und IT-Branche. Sie liefern die strategisch bedeutsamen Ressourcen zur technischen Sicherung der amerikanischen Vormachtstellung in der Welt. Natürlich haben alle diese Bereiche Konkurrenz, zumindest im Ausland, aber die inländischen Anbieter werden politisch gestützt. Hier akzeptiert man Monopole wie die von Microsoft mit einem Weltmarktanteil von über $90 \%$ an der PC-Betriebssoftware, weil sich der Konzern im Gegenzug bei der Vernetzung mit den US-Geheimdiensten (z.B. Übergabe der Verschlüsselungscodes an die NSA etc.) erkenntlich zeigt, was ebenso für Google u.a. gilt.

Bemerkenswert ist weiterhin, dass sich sehr renditestarke Großkonzerne gerade in kleinen Staaten bilden konnten, die nicht nur nationale Marktführer sind, sondern sogar zu den international führenden Unternehmen gehören, wie z.B. die dänische Großreederei Maersk, der Schweizer Konzern Nestlé oder die Bierkonzerne Carlsberg in Dänemark, Heineken in Holland und Interbrew in Belgien. Auch in diesen Ländern gibt es Kartellverbote und Preisabsprachen sind nicht erlaubt. Großzügiger ist man da schon in der Frage der Marktbeherrschung. Diese wird in Deutschland bei 30\% angenommen; der Marktanteil der drei großen Bierkonzerne in ihren Heimatländern liegt z.T. deutlich über 50\%. Die darüber erzielbaren Extraprofite werden toleriert, wenn sie in die internationale Expansion investiert werden. Damit wächst schließlich gleichfalls die am Konzernsitz abzuführende Steuersumme. Und das liegt wiederum im nationalen Interesse.

Auf diese Weise beherrschen etwa drei Konzerne den europäischen Biermarkt, ausgenommen den von England und Deutschland. In Deutschland behaupten sich noch immer gut 1.200 zumeist mittelständische Brauereien auf den überwiegend regionalen Märkten. Sie müssen sich in der Regel mit 2-4\% Umsatzrendite zufrieden geben, während die Shareholder bei den internationalen Konzernen wenigstens das Drei- bis Vierfache erwarten. Um das zu erreichen, verwenden sie billigere Rohstoffe, rationalisieren und zentralisieren den Produktionsprozess, drücken die Löhne und heben die Preise: das klassische Profitsteigerungsprogramm. Der Bierpreis ist in Deutschland denn auch ca. 20\% niedriger als z.B. in Frankreich. Die internationalen Bierkonzerne verzichten auf eine breite Marktpräsenz in Deutschland, weil ihnen die Markteintrittskosten zu hoch sind.

Ähnlich sind die Verhältnisse beim Lebensmitteleinzelhandel in Deutschland. Hier hat sich Walmart, der größte Einzelhandelskonzern mit weltweit 2 Mio. Beschäftigten, 2006 nach einem Milliardenverlust wieder aus dem Markt zurückgezogen. Das Unternehmen kam mit den harten Konkurrenzbedingungen nicht zurecht. Während in den USA mit quasi monopolistischen Anbieterstrukturen für Walmart die Gewinnmarge ca. $6 \%$ beträgt, werden in Deutschland unter den Discountern nur $2 \%$ erreicht. Weil das eingesetzte Kapital in dieser Branche häufig umgeschlagen wird, reicht die niedrige Marge allerdings, um die Brüder Aldi oder die Familie Schwarz (Lidl) zu Milliardären zu machen. Gegenüber den 
Lebensmittelproduzenten stellen die Discounter zusammen mit kleineren Ketten wie Netto, Norma oder Penny sowie den großen Supermarktketten wie Edeka oder Rewe ein Nachfrageoligopol dar, das erheblichen Druck auf die Erzeugerpreise ausübt. Die verlangten Rabatte führen bei den Produzenten zu enormen Rationalisierungen und Lohnsenkungen. Die relativ niedrigen Lebensmittelpreise in Deutschland werden durch niedrige Einkommen in der Landwirtschaft, bei den Lebensmittelproduzenten und im Einzelhandel erkauft.

Betrachtet man die Entwicklung von mergers \& acquisitions in Deutschland, so haben sich diese Tendenzen in den letzten zwei Jahrzehnten in beiden Richtungen beschleunigt: Zusammenschlüsse erfolgen schneller und häufiger als früher, ebenso rasch zerfallen aber auch viele von ihnen. Die Monopolkommission - ein seit 1974 bestehendes Beratungsgremium der Bundesregierung, das zweijährlich Gutachten zum Stand der Konzentration vorlegt stellte 2010 in einem Überblick zu deren Entwicklung in den Jahren von 1978 bis 2008 folgende Veränderungen fest: (1) 1978 betrug der Anteil der Beschäftigten in den 100 größten deutschen Unternehmen 17,4\%, 2008 sind es demgegenüber nur noch 13,3\%. (2) 1978 betrug das Geschäftsvolumen der 50 umsatzstärksten deutschen Unternehmen $31 \%$ aller Industrieunternehmen, 2008 nach größeren Schwankungen, fast ebenso viel, nämlich $31,6 \%$. (3) In anderen Branchen zeigten sich im Vergleich dazu starke Abweichungen: bei Verkehrsund Dienstleistungsunternehmen vereinigten die zehn größten Unternehmen 1994 15,3\% der Umsätze, 2008 aber nur noch $12,8 \%$ (hier schlägt der Wandel der Marktstrukturen bei Post, Flug- und Schienenverkehr zu Buche); demgegenüber ist die Konzentration in anderen Branchen deutlich gestiegen. Bei den Kreditinstituten betrug der Anteil der zehn größten Unternehmen 1978 37,3\% der Umsätze, 2008 aber 50,1\%; ähnlich bei Versicherungen, bei denen sich der Anteil der Umsätze der zehn größten Unternehmen 1990 auf 41,4\%, 2008 aber auf $62,6 \%$ belief.

Diese schlaglichtartigen Befunde weisen auf grundlegende Veränderungen hin. Die „Deutschland AG“ der ersten Jahrzehnte der Bundesrepublik, mit ihrer intensiven und meist auf Langfristigkeit angelegten Verflechtung von Industriekonzernen und großen Banken, gehört der Vergangenheit an. Ihre Auflösung wurde unter anderem durch ein Gesetz der Rot-Grünen Regierung von 2000 befördert, wonach der Verkauf von Unternehmensbeteiligungen seither steuerfrei blieb. Die erhoffte Entflechtung entpuppte sich dennoch vielfach als Scheinerfolg, da mittlerweile andere Unternehmen auf den Plan getreten sind, deren Geschäftsmodell aus dem Kaufen und Verkaufen von Unternehmen besteht. Private equity firms oder hedge fonds haben unter anderem deutsche Firmen wie Grohe, Siemens-Nixdorf oder Rheinmetall aufgekauft oder sich an ihnen beteiligt und dabei kurzfristige Renditeziele insbesondere auf Kosten der Mitarbeiter verfolgt.

Auf internationaler Ebene haben zuletzt Konzentrationsprozesse auf den Rohstoffmärkten Aufsehen erregt. Außer bei denen für Öl und Erdgas, wo sich ihr Einfluss schon deutlich verringert hat, sind die wichtigen Rohstoffmärkte noch fest in der Hand westlicher Konzerne. Bei Erzen und Mineralien beherrschen vier große Unternehmen den Weltmarkt (BHP Billiton, Vale, RioTinto und Glencore Xstrata), was nicht nur auf ein nachfrageinduziertes Wachstum, sondern auch auf eine lange Reihe von Aufkäufen und Fusionen zurückzuführen ist. $\mathrm{Zu}$ letzt hatten sich Glencore, der weltgrößte Rohstoffhändler und der Bergbaukonzern Xstrata zusammengeschlossen, beide im 
Schweizer Niedrigsteuer Kanton Zug ansässig. Die internationale Unternehmensstrategie folgt stets dem gleichen Muster. Die Zentrale bzw. die Firmenholding hat ihren juristischen Sitz in einem Niedrigsteuergebiet. Am Produktionsstandort in schwachen Drittwelt-Staaten wird unter häufiger Verletzung international gültiger Umwelt-, Gesundheits- und Arbeitsstandards zu Niedriglöhnen und mit minimalen Investitionen gefördert. Das Material wird dann so billig an die Zentrale verkauft, dass kaum Gewinn bzw. Verluste und damit auch keine Steuern im Förderland anfallen, während die Zentrale es zum Weltmarktpreis verkauft und den maximierten Gewinn einstreicht. Gern werden noch Zwischenhandelsplätze auf den British Virgin Islands, Bermuda, Jersey etc. eingeschaltet, um durch diese Rochaden eine maximale Steuervermeidung und möglichst unbeschränkte Profitaneignung zu ermöglichen. Für Glencore hat die NGO Erklärung von Bern eine kritische Analyse dazu vorgelegt. Nach Schätzungen des Finanzexperten Raymond W. Baker betrugen die Verluste der betroffenen Entwicklungsländer durch Steuerminimierung im Jahr 2005 mehr als das Doppelte der gesamten in diesem Jahr geleisteten internationalen Entwicklungshilfe von 129 Mrd. Dollar. Als Glencore, das bis dahin dem Management gehörte, im Mai 2011 eine Minderheitsbeteiligung an der Börse verkaufte, entsprach das Aktienpaket, das Glencore-ChefGlasenberg hielt, einem Wert von 8,2 Mrd. Franken, was die Hälfte des BIP von Sambia ausmacht, dessen Kupferminen Glencore seit Jahren ausplündert (Spiegel-Online v. 24.9.2011).

Mergers \& acquisitions gelten führenden Manager-Kreisen nach wie vor als unumgänglich und erfolgversprechend - solange man sie richtig angeht. In der öffentlichen Diskussion wurde „Macht“ seither als Problem nur noch bei staatlichen Monopolen ausgemacht, die es - wie bei Post und Bahn - zu zerschlagen und zu privatisieren galt. Für private Unternehmen erscheint eine derartige Frage jedoch höchstens wie ein schwaches Echo aus einer fernen Welt. Wenn diese eine überragende Position gegenüber Arbeitnehmern, Kunden, Lieferanten oder politischen Körperschaften einnehmen, so sind sie "gut aufgestellt" alles übrige soll sich durch „Wettbewerbspolitik“ und die „Öffnung der Märkte“ erledigen. Angesichts verstärkter internationaler Konkurrenz wird Größe als einzig praktikables Mittel gesehen, um sich auf globalen Märkten zu behaupten. Die in früheren Jahrzehnten, etwa auch in dem führenden deutschen Wirtschaftsmagazin Wirtschaftswoche, noch rege geführte Diskussion zum möglichen gesamtwirtschaftlichen und -gesellschaftlichen Nutzen, aber eben auch Schaden von wachsender Konzentration ist mittlerweile ins Hintertreffen geraten und weitgehend durch die betriebswirtschaftliche Perspektive des best practice ersetzt worden.

Der Mainstream der Wirtschaftswissenschaften hält unbeirrt daran fest, dass große Unternehmen generell als wirtschaftlich überlegen gelten müssen: mit ihren economies of scale und ihren economies of scope würden sie gegenüber kleinen und mittleren Unternehmen schlagende Effizienzvorteile aufweisen. Problematisiert wird ihr Wirken allenfalls, wenn sie eine marktbeherrschende Stellung einnehmen oder mit anderen Oligopolisten Preisabsprachen treffen.

Da waren die Wirtschaftswissenschaften schon einmal sehr viel weiter. Nach der historischen Katastrophe des Zweiten Weltkriegs, zu dem die bereitwillige Kooperation von großen Konzernen wie den IG Farben oder der hochkonzentrierten Stahl- und Elektroindustrie mit der nationalsozialistischen Regierung entscheidend beigetragen hatte, sahen Theoretiker und 
Praktiker der Sozialen Marktwirtschaft in der Nachkriegszeit die Verhinderung von Konzentration als eine wichtige Aufgabe an. Das ab 1958 gültige Gesetz gegen Wettbewerbsbeschränkungen entsprach allerdings nur zum Teil ihren Vorstellungen, die in die Richtung einer durchgängig klein- und mittelständisch strukturierten Unternehmenslandschaft gingen, was vielen Zeitgenossen und insbesondere den führenden Kreisen der Wirtschaft als anachronistische Bilderbuchidylle erschien. Das Gesetz verbot horizontale und vertikale Kartellierungen, ließ $\beta$ jedoch zahlreiche Ausnahmen zu und enthielt nur schwache Instrumente zur Kontrolle von Fusionen. Dennoch blieb das Thema Konzentration in der Öffentlichkeit und in der Wissenschaft auf der Tagesordnung. Ab 1962 bestand an der FU Berlin ein Institut für Konzentrationsforschung, dessen Leiter Helmut Arndt in den folgenden Jahren zahlreiche Studien zur Entwicklung der Konzentration in der Wirtschaft vorlegte und „Wirtschaftliche Macht" in einem sehr viel umfassenderen Verständnis thematisierte, als es zu seiner Zeit, und vielfach auch später, üblich war.

Damals beschäftigten sich auch Linke gerne mit diesem Themenkomplex - so etwa die Assistenten Arndts, die 1970 gegen ihren Chef rebellierten und die marxistische Konzentrationstheorie in den Mittelpunkt stellen wollten, was letztlich zum Rücktritt Arndts von der Leitung des Instituts und zu dessen Umbenennung führte. Die Assistenten-Revolte sollte sich als PyrrhusSieg erweisen, denn damit wurde ein anerkannter und einflussreicher Kritiker der herrschenden wirtschaftswissenschaftlichen Theorie und der wirtschaftspolitischen Praxis tendenziell marginalisiert.

Arndt wandte sich gegen die Ausblendung von Macht in der neoklassischen Modellwelt und zeigte, dass Unternehmen als Aktionsparameter keineswegs nur über
Preise und Mengen verfügten. So könnten sie, wenn ihre Marktstellung es ihnen erlaubte, unter anderem: Produkte standardisieren oder den Kundendienst verschlechtern, ohne die Kosteneinsparungen an ihre Kunden weiter zu geben; die Qualität von Produkten verschlechtern und deren Lebensdauer verringern, um die Nachfrage zu erhöhen; die eigene Lagerhaltung reduzieren, was die Abnehmer zu erhöhter Lagerhaltung zwingt; ihren Kunden überhöhte Zinsen oder Gebühren auferlegen. Wirtschaftliche Macht könne sich auch in der Dominanz von Unternehmen gegenüber anderen Unternehmen äußern, die von ihnen abhängig sind. Die Zahl der Beherrschungsverhältnisse übertreffe insofern um ein Vielfaches die Zahl der Monopole. Im übrigen habe die Größe von Unternehmen entscheidende Bedeutung für ihre Position gegenüber staatlichen Stellen: Im Fall von Krisen seien große Unternehmen in der Lage, mit Hinweis auf ihre volkswirtschaftliche Bedeutung Subventionen einzufordern; sie verfügten über Wissen, das sie als Insiderinformationen nutzen konnten; das Depotstimmrecht verleihe den Vorständen großer Banken die Möglichkeit, sich wie allmächtige Despoten zu gerieren und die Kontrolle durch Aufsichtsräte zu unterlaufen; international tätige Unternehmen könnten ihre Gewinne und Verluste in die Länder ihrer Wahl verlagern und auf diese Art Steuern sparen und schließlich könnten große Unternehmen gegenüber staatlichen Instanzen als pressure groups auftreten und Entscheidungen in ihrem Sinn beeinflussen. Arndts Fazit: „In einer Welt, in der die wirtschaftliche Macht noch ungebändigt ist, sind auch die Menschenrechte nicht oder nur unvollkommen realisiert." (Wirtschaftswoche Nr. 2/1979: 72). Dieser wache Blick für die Vielfalt der Erscheinungsformen wirtschaftlicher Macht zeichnete einen Ökonomen aus, der aus linker Sicht 
als „bürgerlich“ gelten musste, mit seinen nüchternen Analysen aber die vieler Linken mit ihrer schematischen Fixierung auf die „Macht der Monopole“, (etwa in der Form des "staatsmonopolistischen Kapitalismus"), weit übertraf.

Ähnliche Schematisierungen treten auch heutzutage auf, wenn es um die Rolle des als übermächtiger Dämon porträtierten Finanzkapitals geht. Die in diesem Heft publizierten Artikel sollen eine differenziertere Sicht voranbringen. Mehrere Autor/inn/en setzen sich mit der Frage auseinander, wie sich die wachsende Dominanz der Finanzlogik bei großen Unternehmen auswirkt. Claude Serfati vertritt die Auffassung, dass multinationale Unternehmen eine besondere Form des Finanzkapitals darstellen, wobei eine zunehmende Diskrepanz zwischen deren Buch- und Börsenwerten festzustellen ist. Julian Müller zeigt, dass sich die schon häufig diagnostizierte Finanzialisierung darin niederschlägt, dass viele Unternehmen mittlerweile von den Erwartungen der Investoren und den Ratings der großen Agenturen abhängen, so dass sie ihre Strategien weniger als früher an der Optimierung der industriellen Produktion durch Forschung und Entwicklung ausrichten, sondern kurzfristig zu erzielende Erfolge mit mergers and acquisitions anstreben, um sich Eigentumsrechte und Lizenzen anzueignen. Beide Aufsätze schließen kritisch an die Marxschen Analysen im Kapital an. Diese grundsätzlichen Überlegungen werden dann in mehreren Fallstudien vertieft. Jürgen Kädtler verfolgt die Entwicklung der großen Drei in der Chemieindustrie, die als Resultat der Entflechtungen in der Nachkriegszeit entstanden und für die lange Zeit das Modell der "Verbundchemie“ galt. Die beiden übrig gebliebenen Konzerne entwickelten höchst unterschiedliche Wege des Umgangs mit der Finanzialisierung, was auch mit dem Einfluss von Vertretern der
Belegschaft zu tun hat. Barbara Brandl untersucht den globalen Saatgutmarkt und weist auf Widerständigkeiten und Brüche der Konzentrationsbewegung hin, da Patente nicht immer den Königsweg darstellen, sondern sich auch das inkorporierte Wissen der Züchter/innen als entscheidend erweisen kann. Christian Zeller analysiert die oligopolistische Rivalität in der Pharma- und Biotechindustrie, wobei sowohl Prozesse der vertikalen und horizontalen Desintegration wie eine zunehmende Steuerungsmacht der Großen der Branche zu beobachten sind, die gleichwohl unterschiedliche Strategien einschlagen können. Schließlich soll der Blick über Westeuropa und die USA hinaus auf die Zustände bei einem der neuen global player gerichtet werden. Sarah Nagel widmet sich in ihrem Beitrag den in den letzten Jahren international immer stärker auftretenden chinesischen Staatskonzernen.

Außerhalb des Schwerpunkts analysiert Horst Heitmann die Außenpolitik des gerade wieder gewählten Barack Obama. Der in Europa noch immer als Anti-Bush betrachtete Obama weist in der Außenpolitik eine Vielzahl von Kontinuitäten mit Bush junior auf und es besteht wenig Hoffnung, dass sich in seiner zweiten Amtszeit daran etwas ändern wird. Mit dem zweiten Beitrag außerhalb des Schwerpunktes schließen wir an die beiden PROKLA-Hefte zur Gesellschaftstheorie an (Nr. 165 Gesellschaftstheorie im Anschluss an Marx, Dezember 2011, Nr. 167 Perspektiven der Gesellschaftskritik heute, Juni 2012). Loïc Wacquant analysiert die historische Anthropologie des gegenwärtigen, neoliberalen Gesellschaftsgefüges in Abgrenzung sowohl zu rein ökonomischen Erklärungsansätzen als auch zu den an Foucault anschließenden Gouvernementalitätsstudien. Zugleich zieht er damit ein Resümee seiner Forschungen der letzten beiden Jahrzehnte. 